\title{
Bidirectional association between knee osteoarthritis and depressive symptoms: evidence from a nationwide population-based cohort
}

\author{
Han Lu' ${ }^{1}$ Limin Wang ${ }^{1,2}$, Weijiao Zhou ${ }^{3}$, Shida Jin ${ }^{1}$, Hongbo Chen ${ }^{1,4}$, Yi Su$^{5}$, Nan Li ${ }^{6}$ and Shaomei Shang ${ }^{1 *}$
}

\begin{abstract}
Background: Both knee osteoarthritis (KOA) and depressive symptoms (DS) are major public health issues affecting the quality of life. This study aimed to examine the association between KOA and DS.

Methods: Data were gathered from the China Health and Retirement Longitudinal Study in 2011-2015 which surveyed middle-aged to elderly individuals and their spouses in 28 provinces in China. An adjusted Cox proportional hazards regression model was used to estimate hazard ratios (HRs).

Results: The analysis for baseline KOA and the subsequent risk of DS was based on 2582 participants without baseline DS. During the follow-up, KOA patients were more likely to have DS than non-KOA participants (adjusted $H R=1.38: 95 \% \mathrm{Cl}=1.23$ to 1.83). The analysis for baseline DS and the subsequent risk of KOA was based on 4293 participants without baseline KOA, those with DS were more likely to develop KOA than non-DS participants (adjusted $\mathrm{HR}=1.51: 95 \% \mathrm{Cl}=1.26$ to 1.81 ). Subgroup analysis showed sex and age had no significant moderating effect on the KOA-DS association.
\end{abstract}

Conclusions: Our results provide evidence that the association between KOA and DS is bidirectional. Therefore, primary prevention and management of KOA and DS should consider this relationship.

Keywords: knee osteoarthritis, depressive symptoms, longitudinal studies, proportional hazards models

\section{Background}

Knee osteoarthritis (KOA) and depressive symptoms (DS) are major physical health and mental health problems affecting the quality of life of older people, respectively. Specifically, KOA is one of the leading causes of disability in the elderly [1], accounting for approximately $85 \%$ of the burden of osteoarthritis (OA) worldwide [2]. Persons with KOA commonly experience pain, aching, stiffness, and associated functional

\footnotetext{
*Correspondence: shangshaomei@126.com

1 Peking University School of Nursing, No.38 Xueyuan Road, Haidian District, Beijing, China

Full list of author information is available at the end of the article
}

loss [3]. Given the increased life expectancy and ageing of the global population, the prevalence, incidence, and years lived with disability due to KOA are expected to continue increasing in most countries [4]. What's more, there is no known cure for KOA [5]. Depressive disorders ranked 13th in the causes of global disabilityadjusted life-years (DALYs) in the latest Global Burden of Disease Study [6]. Multiple studies have found the aggregation of DS in KOA patients [7-11]. Combined depression/DS could aggravate patients' pain and disability, reduce treatment adherence, and increase the healthcare burden [12-14]. Agarwal P et al. found that OA patients with depression could increase the annual 
medical expenses by nearly US $\$ 4400$ [15]. Therefore, it is necessary to pay more attention to the KOA with depression/DS as a major public health issue.

There are two possible explanations for the aggregation of DS in KOA patients: patients with KOA have a high risk to develop depression, or depressed persons are more likely to develop KOA. This has not been determined yet. On the one hand, DS is reportedly highly prevalent among KOA patients, and KOA has been cited as a risk factor for developing DS [711]. On the other hand, some studies have shown that patients with DS are more likely to develop arthritis $[11,16]$. Although many studies have explored the single-directional relationship between KOA and DS, whether the relationship between KOA and DS in both directions coexists in the same group is less verified. Moreover, most of the current studies were based on population in developed countries, and there is a lack of evidence from China.

To fill this gap, our study aimed to use data from a middle-aged and elderly cohort to determine whether the association between KOA and DS is bidirectional and the strength of association. Firstly, we analyzed the effect of baseline KOA on the incidence of subsequent DS; secondly, we analyzed the effect of baseline DS on the incidence of subsequent KOA. Besides, we explore the strength of these effects at different age, sex groups.

\section{Methods}

\section{Participants}

Data for this study were gathered from the baseline survey in 2011 and two follow-up surveys in 2013 and 2015 of the China Health and Retirement Longitudinal Study (CHARLS). The CHARLS national survey used a multistage sampling strategy covering 28 provinces, 150 counties or districts, and 450 villages or urban communities in China. Successfully interviewed 17,708 individuals from 10,257 households, representing the middle-aged and elderly people in China in general [17]. In the first analysis to predict the incidence of DS, participants were eligible if (i) they were $\geq 45$ years old; (ii) they did not have DS at baseline; (iii) they were followed up for $\geq 1$ year, and status of DS during follow-up was recorded; (iv) they had complete baseline information (including KOA status and covariates). The second analysis to predict the incidence of KOA included participants if (i) they were $\geq 45$ years old; (ii) they did not have KOA at baseline; (iii) they were followed up for $\geq 1$ year, and status of KOA during follow-up was recorded; (iv) they had complete baseline information (including DS status and covariates).

\section{Measures}

KOA onset

All participants had a face-to-face household interview using a structured questionnaire. According to the definition in a previous study [18], KOA was identified by selfreported both physician-diagnosed osteoarthritis and the presence of concurrent knee pain. Physicians who made the diagnosis include internists, rheumatologists, orthopedic surgeons and doctors in Chinese medicine, and the diagnosis is often reached with the aid of X-rays [18, 19].

\section{Depressive symptoms}

DS was measured by the Chinese version of the Center for Epidemiologic Studies Depression (CES-D), a 10-item questionnaire with good reliability and validity (possible range, $0 \sim 30$ ) [20]. The presence of DS was defined by a CES-D score of 10 or higher [20].

\section{Covariates}

Covariates in the analysis included sociodemographic characteristics, lifestyle, and health-related factors at baseline. All the variables were obtained from the 2011 CHARLS survey. As described in previous studies [8, 21, 22], the following factors were selected as covariates: a) sociodemographic characteristics: age, sex, marital status, education level, yearly income [23], and residence (urban, rural); b) lifestyle: smoking status, drinking status, length of sleep, physical activity (calculated using metabolic equivalent multiplied by activity weekly duration level) $[24,25]$; c) health-related factors: whole health and childhood health status (a 5-point Likert scale, where higher scores mean better health), body mass index (BMI), the number of difficulties with activities of daily living (ADL) [26] and instrumental activities of daily living (IADL) [27]. the number of non-communicable diseases (NCDs), experienced major accidental injury or not, and C-reactive protein (CRP) [21]. Except for $\mathrm{BMI}$ and CRP were measured on site, all variables were obtained from self-reported questionnaire data, and the detailed information related to how each covariate was assessed and the used tools is presented in Supplementary Table S1.

\section{Statistical analysis}

Continuous variables are reported as mean \pm standard deviation (SD), while categorical variables are expressed using proportions. We used t-tests and chi-square tests to analyze differences among groups. We used KaplanMeier plots to show the incidence of DS by KOA or non-KOA, and the incidence of KOA by DS or not. Logrank tests were used to compare the differences between groups. The duration of follow-up was defined as the date 
of the baseline interview to either the date of DS/KOA onset or the date of the last interview. The incidence density of KOA and DS was estimated by the number of incident patients divided by the number of person-years accumulated in the population. The proportional hazards assumption was checked using the Schoenfeld residuals test. As the test results were reported as $P>0.05$, the proportional hazard assumptions were satisfied. Cox proportional-hazard regression was used to calculate the crude and adjusted hazard ratios (HRs) with corresponding 95\% confidence intervals (CIs). Furthermore, we performed stratified Cox regression analysis to examine the modifying effect of age ( $<65$ years and $\geq 65$ years), sex on the bidirectional KOA-DS associations. All tests were 2 -tailed, and the significance level was set to $P<0.05$. Stata 15.1 was used for analysis.

To check whether the analyzed sample was representative of the entire population, we described the characteristics of the CHARLS population and the middle-aged and elderly KOA and DS incident cohorts, as well as the distribution of missing values (Supplementary Table S2). Considering the potential bias caused by excluding missing data participants, we performed sensitivity analyses comparing crude HRs between the analytical sample and the incident cohorts with missing values (Supplementary Table S3 and S4).

\section{Results}

The cohort analysis for baseline knee osteoarthritis and the subsequent risk of depressive symptoms

The analysis for the risk of DS with KOA was based on 2582 participants free from DS at baseline (See Fig. 1 for the selection process). Table 1 shows the baseline characteristics of the KOA and non-KOA participants. KOA was found more frequently among women, rural residents, those with at most primary education and lowincome persons. Other characteristics associated with KOA were fewer sleep hours, worse whole health, worse childhood health, higher BMI, more difficulties with $\mathrm{ADL}$ and IADL, more NCDs, and more accidental injury $(P<0.05$ for all these variables).

During the four-year follow-up, 756 individuals (29.3\% of the baseline population) developed DS, with an incidence of 8.22 new cases per 1000 person-months. Specifically, the incidence density of DS in KOA patients was 15.48 per 1000 person-months, compared with 7.73 per 1000 person-months among non-KOA participants (Table 2). The Kaplan-Meier survival analysis showed that participants with KOA had an increased risk of DS (Fig. 2). Cox proportional hazards regressions further demonstrated the effect of KOA on DS. Participants with KOA were more likely to develop DS than those without KOA (HR 2.05; 95\% CI=1.65 to 2.56). After adjusting for all potential confounders, this effect was still statistically significant. Among all subgroups, persons with KOA had a higher incidence of DS, with females $<65$ having the highest incidence (Table 2). The stratified analysis found that age and sex did not modify the association between baseline KOA and incident DS, the $P$-value for KOAsex interaction and KOA-age interaction was 0.993 and 0.474 , respectively.

\section{The cohort analysis for baseline depressive symptoms and the subsequent risk of knee osteoarthritis}

The parallel analysis of DS predicting KOA onset was based on 4293 participants free from KOA at baseline

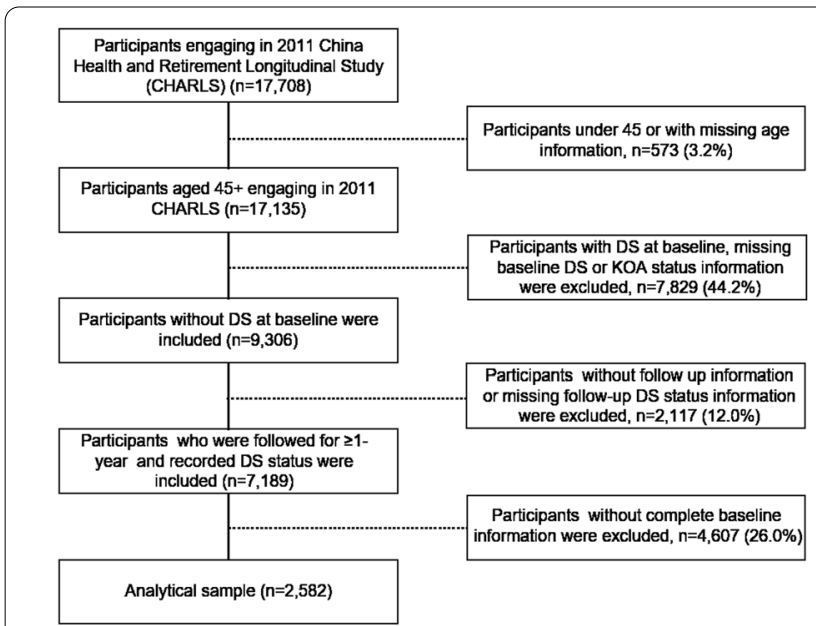

(a) Flowchart of the selection of sample in the cohort analysis for baseline knee osteoarthritis and the subsequent risk of depressive symptoms.

Fig. 1 Flowchart of the selection of sample

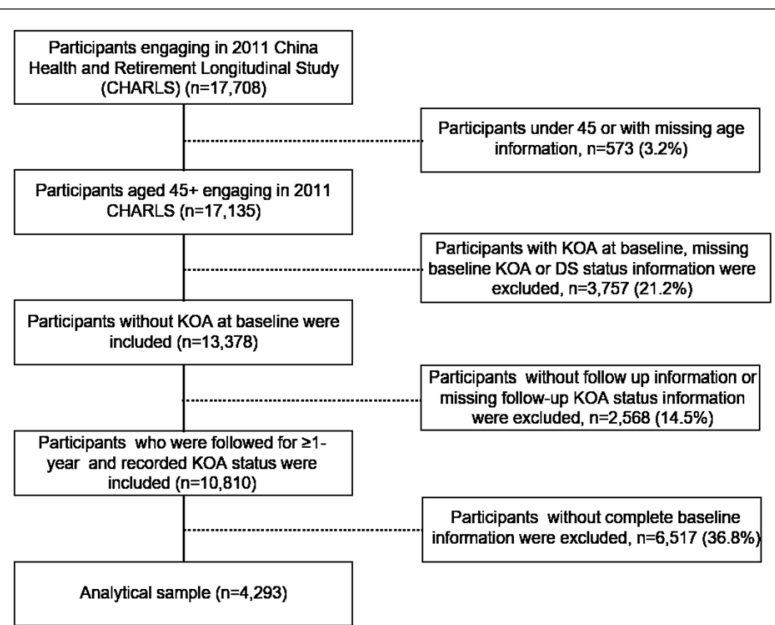

(b) Flowchart of the selection of sample in the cohort analysis for baseline depressive symptoms and the subsequent risk of knee osteoarthritis. 
Table 1 Baseline Characteristics of Participants Included in the Analysis

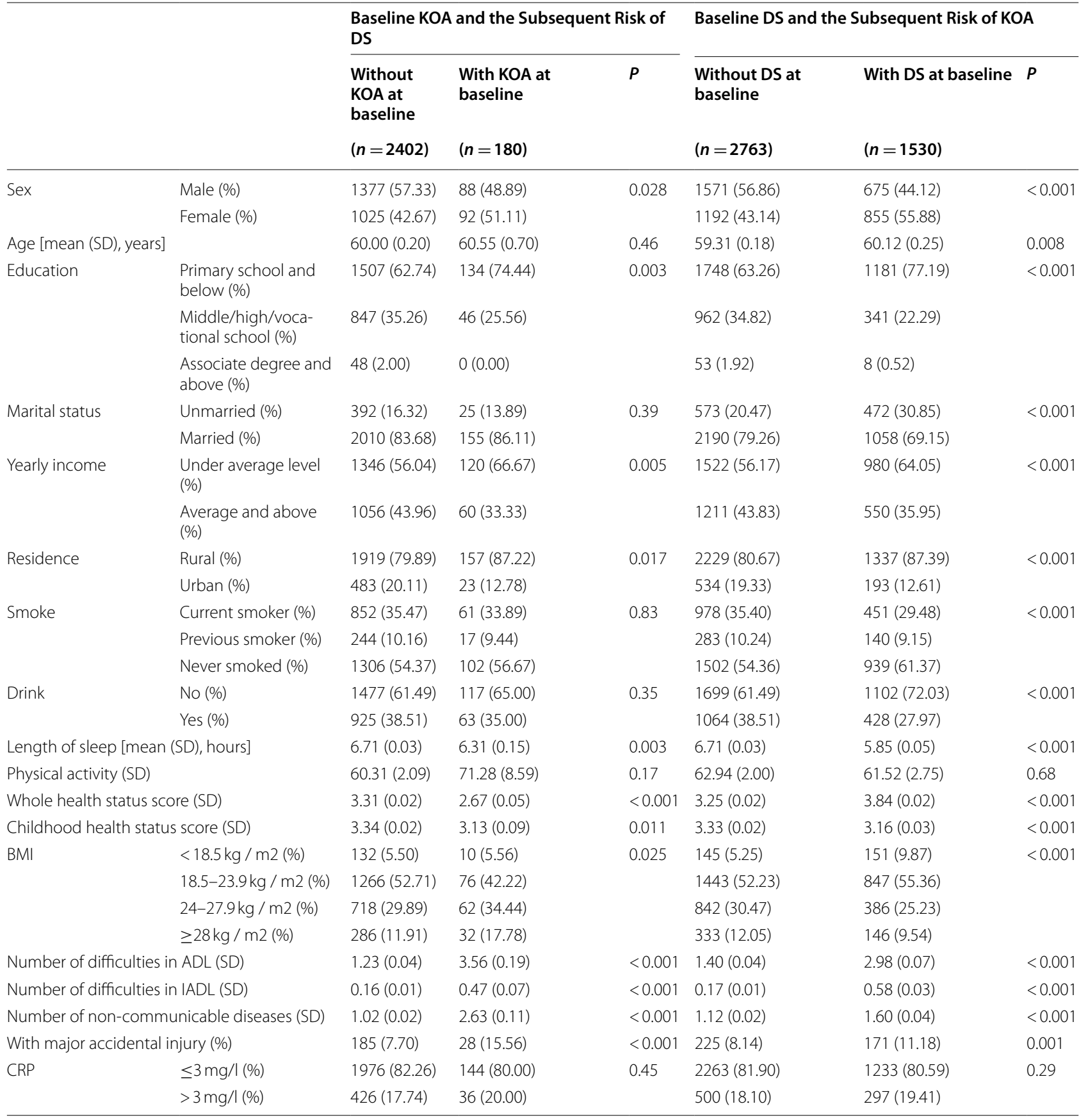

$B M I$ body mass index, $A D L$ activities of daily living, IADL instrumental activities of daily living, CRP C-reactive protein

(see Fig. 1 for the selection process and Table 1 for baseline characteristics). Compared with those without DS at baseline, participants with DS had a higher proportion of females, aged 65 and older, less educated, unmarried, low-income population, and rural residents. On the other hand, there were fewer smokers and drinkers among patients with DS, and their health status was worse (embodied in the length of sleep, overall health, childhood health, BMI index, difficulties with ADL and IADL, number of NCDs, and injury events).

Over the four years, 469 individuals (10.9\%) developed KOA, with an incidence of 2.40 new cases per 1000 person-months. In the group of individuals with DS, the incidence density of KOA was 3.89 per 1000 
Table 2 Incidence and Hazard Ratios for Depressive Symptoms in Association with Knee Osteoarthritis, Overall and Stratified by Age and $\operatorname{Sex}(N=2582)$

\begin{tabular}{|c|c|c|c|c|c|c|}
\hline & With KOA at baseline & & Without KOA at base & & Adjusted HR* & $P$ \\
\hline & $\begin{array}{l}\text { Event/at risk } \\
\text { (n / person-months) }\end{array}$ & $\begin{array}{l}\text { Incidence rate per } \\
1000 \text { person-months }\end{array}$ & $\begin{array}{l}\text { Event/at risk } \\
\text { (n / person-months) }\end{array}$ & $\begin{array}{l}\text { Incidence rate per } \\
1000 \text { person-months }\end{array}$ & & \\
\hline Overall & $90 / 5814$ & 15.48 & $666 / 86,164$ & 7.73 & $1.38(1.07-1.78)$ & $<0.001$ \\
\hline Age $<65$ & $64 / 4155$ & 15.40 & $481 / 63,737$ & 7.55 & $1.53(1.13-2.07)$ & 0.007 \\
\hline Male & $23 / 2075$ & 11.08 & $218 / 36,283$ & 6.01 & $1.5(0.91-2.46)$ & 0.11 \\
\hline Female & $41 / 2080$ & 19.71 & $263 / 27,454$ & 9.58 & $1.63(1.10-2.42)$ & 0.015 \\
\hline Age $\geq 65$ & $26 / 1659$ & 15.67 & $185 / 22,427$ & 8.25 & $1.29(0.81-2.06)$ & 0.29 \\
\hline Male & $13 / 939$ & 13.84 & $101 / 14,641$ & 6.90 & $1.3(0.65-2.58)$ & 0.46 \\
\hline Female & $13 / 720$ & 18.06 & $84 / 7786$ & 10.79 & $1.1(0.54-2.22)$ & 0.79 \\
\hline
\end{tabular}

The $P$-value for KOA-sex interaction was 0.993 . The $P$-value for KOA-age interaction was 0.474 .

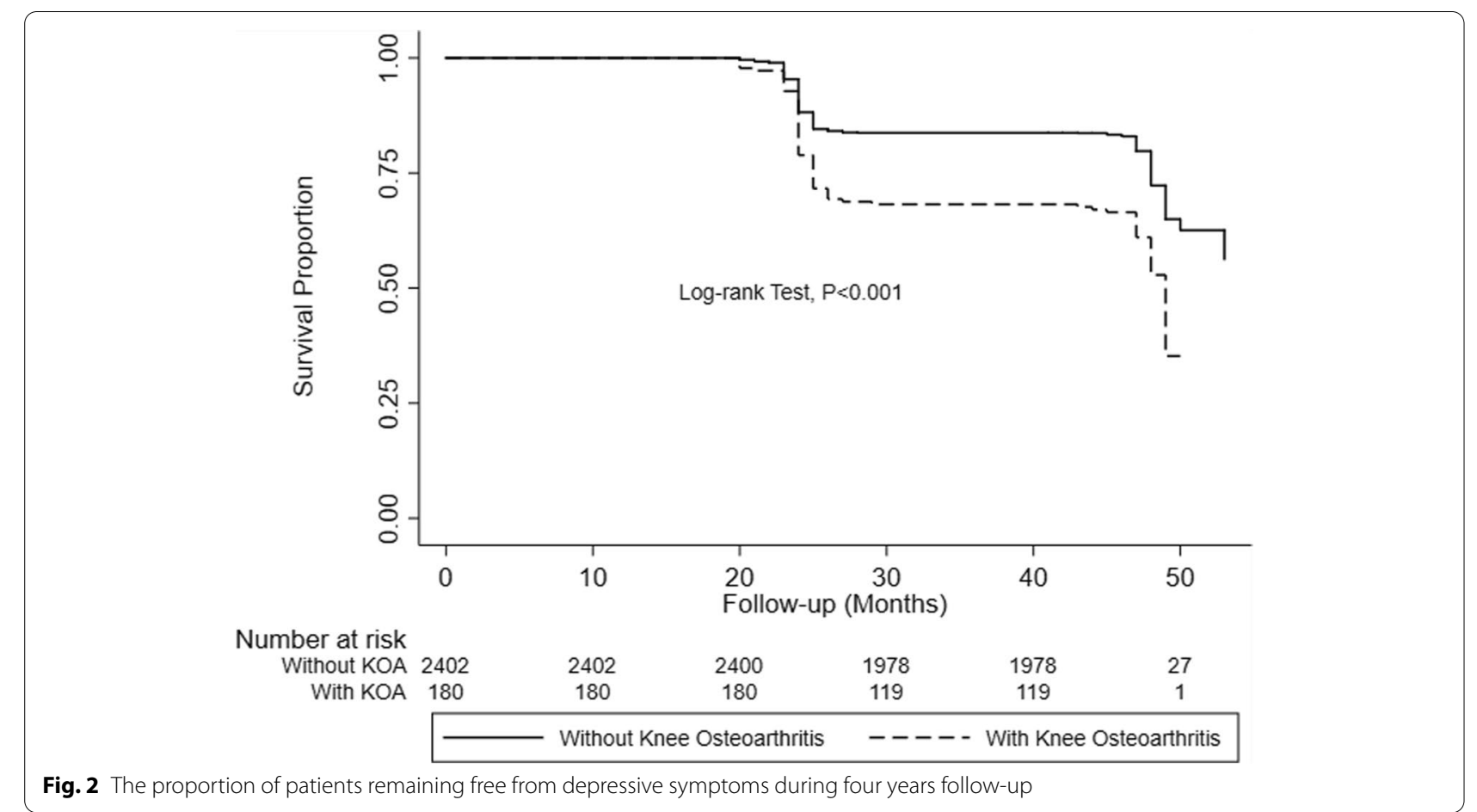

person-months, which was 2.45 times that of the control group (Table 3). Kaplan-Meier survival analysis and Cox proportional hazards regression suggested that patients with DS had a higher risk of developing KOA (Fig. 3); crude HR for KOA associated with baseline DS was $2.52(95 \% \mathrm{CI}=2.10$ to 3.03$)$, and the adjusted HR was $1.51(95 \% \mathrm{CI}=1.23$ to 1.84$)$ (Table 3$)$. In the subgroup analysis, regardless of age and sex, the incidence of KOA in patients with baseline DS was higher than in patients without baseline DS, with females aged $<65$ years having the highest incidence rate (Table 3 ). DS-sex interaction and DS-age interaction analysis showed that neither age nor sex mediated the association between DS and KOA.

\section{Discussion}

This national cohort analysis provided compelling evidence that the association between KOA and DS is bidirectional. We found that patients with baseline KOA had a higher incidence of DS and their risk of developing DS within four years was 1.38 times that of persons without baseline KOA. In contrast, a higher incidence of KOA can be identified in individuals with baseline DS. Baseline DS increased the risk of KOA by $51 \%$ over four years 
Table 3 Incidence and Hazard Ratios for Knee Osteoarthritis in Association with Depressive Symptoms, Overall and Stratified by Age and $\operatorname{Sex}(N=4293)$

\begin{tabular}{|c|c|c|c|c|c|c|}
\hline & With DS at baseline & & Without DS at baseli & & Adjusted HR (95\% Cl) & $P$ \\
\hline & $\begin{array}{l}\text { Event / at risk } \\
\text { (n / person-months) }\end{array}$ & $\begin{array}{l}\text { Incidence rate } \\
\text { per } 1000 \text { person-months }\end{array}$ & $\begin{array}{l}\text { Event/at risk } \\
\text { (n / person-months) }\end{array}$ & $\begin{array}{l}\text { Incidence rate } \\
\text { per } 1000 \text { person-months }\end{array}$ & & \\
\hline Overall & $267 / 68,565$ & 3.89 & $202 / 126,723$ & 1.59 & $1.51(1.23-1.84)$ & $<0.001$ \\
\hline Age $<65$ & $192 / 48,068$ & 3.99 & $140 / 91,970$ & 1.52 & $1.62(1.27-2.07)$ & $<0.001$ \\
\hline Male & $68 / 19,965$ & 3.41 & $58 / 50,866$ & 1.14 & $1.64(1.09-2.48)$ & 0.017 \\
\hline Female & $124 / 28,103$ & 4.41 & $82 / 41,104$ & 1.99 & $1.59(1.16-2.17)$ & 0.004 \\
\hline Age $\geq 65$ & $75 / 20,497$ & 3.66 & $62 / 34,753$ & 1.78 & $1.35(0.93-1.96)$ & 0.115 \\
\hline Male & $34 / 10,397$ & 3.27 & $27 / 21,430$ & 1.26 & $1.34(0.74-2.40)$ & 0.33 \\
\hline Female & $41 / 10,100$ & 4.06 & $35 / 13,323$ & 2.63 & $1.31(0.79-2.16)$ & 0.29 \\
\hline
\end{tabular}

The $P$-value for DS-sex interaction was 0.346. The $P$-value for DS-age interaction was 0.325 .

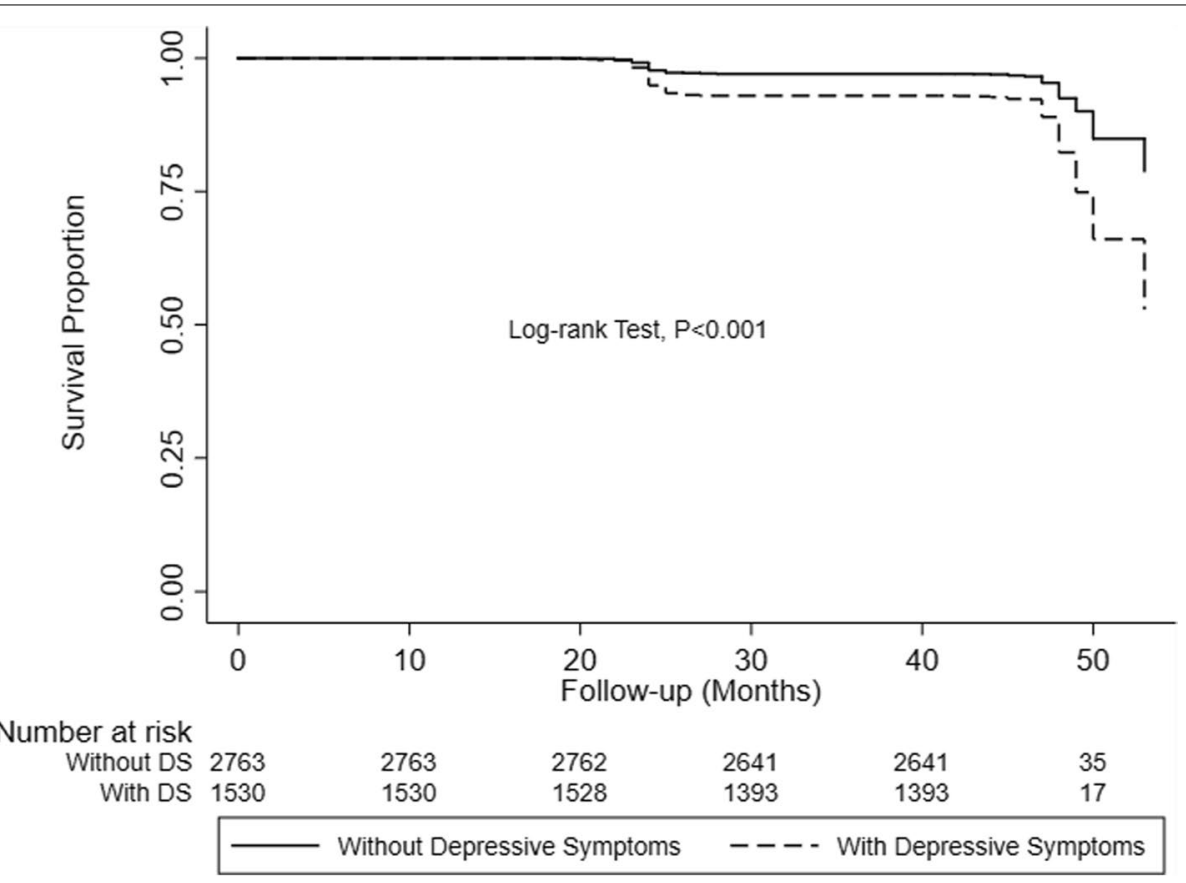

Fig. 3 The proportion of patients remaining free from knee osteoarthritis during four years follow-up

of follow-up. This two-way association was independent of sociodemographic factors and individual lifestyle and health-related factors.

Existing evidence has focused mainly on the risk of depression in KOA patients. A recent meta-analysis demonstrated that approximately $18.5 \%$ of KOA patients also have DS, which is higher than the prevalence in the general population $[9,28]$. Nicola et al. reported that the incidence of DS associated with KOA was 26 per 1000 person-years, KOA patients were more likely to develop DS (odds ratio, $=1.43$ : $95 \% \mathrm{CI}=1.03$ to 1.98 )
[29]. Another study analyzing the association of cancer, cardiovascular disease, diabetes, and OA at baseline with incident depression found that among these four conditions OA had the highest risk for incident depression $(\mathrm{HR}=1.94$ : $95 \% \mathrm{CI}=1.80$ to 2.10$)$ [30]. However, most of the existing research samples have come from high-income countries, whereas our research provides evidence from China. And the incidence of DS related to KOA in China is higher than that reported in previous studies in other countries. There are several studies focusing on the risk of KOA in patients with DS. Previous 
analyses on the relationship between OA and depression showed similar results to ours, that is, the presence of DS could increase the risk of OA $[11,16]$.

The actual mechanisms contributing to the association between KOA and DS have not been well understood yet. Regarding the susceptibility of KOA patients to DS, depression and DS in patients with chronic diseases are usually attributed to the emotional response to the diagnosis of the disease, or the lifestyle limitations due to the disease [31]. Studies have suggested that pain, disability, reduced physical activity, obesity, and sleep disturbance might be important causes for DS among KOA patients $[32,33]$. This study also found that longer sleep duration and better overall health status were important protective factors for developing DS (Supplementary Table S5). As for why depressed patients are prone to KOA, there is currently a lack of relevant research. According to previous studies, depressed individuals are more likely to adopt unhealthy lifestyles, such as unhealthy diets and sedentary behavior, which are risk factors for KOA [34, 35]. Milaneschi $Y$ et al. found that compared with controls [36], patients with depression had more vitamin D deficiency, while evidence showed that low vitamin D level was an important predictor of KOA [37]. Concurrently, research implied that both KOA and depression could be regulated by similar inflammatory immune mechanisms. The production and release of TNF $\alpha, \mathrm{IL}-1 \beta$, IL-6, and IL- 8 have been detected in the synovial fluid, synovial tissue and serum of patients with KOA [38, 39]. These pro-inflammatory factors participate in the pathogenesis of KOA through the hypothalamic-pituitary-adrenal axis, causing crosstalk between the brain and the immune system. On the other hand, these proinflammatory factors are also important factors regulating central pain and depression. Donovan et al. have tested TNF- $\alpha$, IL-6, IL-10, CRP, and other inflammatory factors in patients with depression and healthy controls and found that these indicators were independently related to patients' suicidal ideation [40]. However, the CRP $(\leq 3 \mathrm{mg} / \mathrm{l}$ or $>3 \mathrm{mg} / \mathrm{l})$ included in this analysis was not observed to affect the incidence of KOA and DS (Supplementary Table S5 and S6). Further research considering biological, behavioral, and physiological pathways is needed to understand the mechanisms of this association.

In the subgroup analysis, we found that in all age and sex groups, participants with baseline KOA had a higher incidence of DS, accordingly, participants with baseline DS had a higher incidence of KOA. This result is consistent with our assumptions. As for some subgroups, the risk of DS associated with KOA and the risk of KOA associated with DS were not statistically significant, which may be mainly related to insufficient sample size. Similar to other surveys [3, 41], both KOA and DS are very common among middle-aged and older people, especially among females. However, gender was not identified as a mediator in the relationship between KOA and DS in this research, which is consistent with another longitudinal study [30]. And we found no mediating effect of age, either. A weakened relationship between KOA and DS was observed in the $65+-$ year age group compared to the 45-65-year age group. One reason may be a selection bias in this study. Because people aged $\geq 65$ years are more likely to suffer from long-term KOA or DS, it may be more common to find people with both DS and KOA among those aged $\geq 65$ years. However, these people were excluded, and selected older participants may be less susceptible to the bidirectional relationship between KOA and DS. Beyond that, studies have also offered some explanations. The decreased risk of DS associated with KOA in the elderly group may be because older people pay less attention to the functional impairment caused by KOA than middle-aged people [42], while middle-aged were still juggling many roles (such as family and work responsibilities) and did not expect difficulties with their daily activities. Similarly, the HR for KOA associated with baseline DS was smaller in older adults than in middleaged. Evidence showed that older patients with DS may increase the competing risk of death from other causes $[43,44]$, which masked the incidence of KOA. Also, a previous study revealed that older adults may less likely to report DS [45], and the non-differential misclassification of DS could bias the association towards null.

\section{Implications for mental health nursing}

KOA and DS often coexist in patients, which leads to increased medical costs and a worse prognosis $[9,12,15]$. We need to pay attention to the coexistence of KOA and DS, and explore the association between the two. This study proved a bidirectional association between KOA and DS through cohort analysis, patients with KOA have more DS than the general population, and depressed patients are more likely to develop KOA.

Considering the susceptibility of KOA patients to DS, there is a need to incorporate depression screening, highrisk group prevention, and treatment into the management of KOA patients. The National Institute for Health and Clinical Excellence (NICE) also recommended that patients with OA should strengthen the treatment and management of depression [46]. When facing depression patients, osteoarthritis prevention guidance also needs to be carried out. Exercise therapy as an important nonpharmacological treatment has been found to not only bring specific KOA prevention benefits, but also improve quality of life and psychosocial factors [47, 48]. Previously, it has been found that there is a strong connection 
between coronary heart disease/chronic obstructive pulmonary disease/diabetes and depression/DS, the bidirectional association between KOA and DS once again proves the interactive relationship between physical health and mental health [49-51]. It is necessary to pay attention to the bidirectional association in the practice, and strengthen the communication and cooperation between mental health practitioners and physical health practitioners.

\section{Study strengths and limitations}

The strengths of this study are the longitudinal study design and the use of population-based data that provide sufficient sample size and statistical power, as well as the validated and widely used 10-item CES-D. Nevertheless, this study had several limitations. First, we adopted selfreported physician-diagnosed osteoarthritis and knee pain to determine KOA, with possible chances for misclassification. Second, we analyzed only baseline KOA and baseline DS, that is, without considering any KOA or DS that might emerge during the follow-up period, which may reduce the apparent effects of KOA on the incidence of DS and of DS on the incidence of KOA. But this still does not prevent us from concluding there is a bidirectional relationship between KOA and DS. And there may be bias in the older subgroup analysis by excluding older adults with both KOA and DS at baseline. Third, we excluded participants without complete covariates information which may lead to selection bias, however, the sensitivity analysis showed only small differences of crude HRs between included sample and incident cohorts with missing values. Besides, the database does not contain information about the severity of KOA and treatments information (only includes the degree of pain, but it cannot be included in the analysis because most of the population lacks this data), so we cannot assess the relationship between the severity of KOA or treatments and subsequent DS risk.

\section{Conclusions}

KOA and DS are major problems that plague the health of older adults. Patients with KOA often suffer from DS, which reduces their quality of life and increases the healthcare burden. This large, well-established cohort study provides compelling evidence that the association between KOA and DS is bidirectional. And this association cannot be fully explained by risk factors such as age, sex, BMI, physical activity, sleep time, and disabilities. Our findings remind both mental health practitioners and physical health practitioners to carefully consider this bidirectional association in the primary prevention and management of KOA and DS. When facing patients with KOA or depression/DS, it is crucial to strengthen the screening and treatment of another disease while treating the visiting disease.

\section{Supplementary Information}

The online version contains supplementary material available at https://doi. org/10.1186/s12891-022-05137-8.

Additional file 1. Supplementary materials. Table S1-S6.

\section{Acknowledgements}

Grateful to the Peking University National School of Development and Institute of Social Science Survey for providing the data.

\section{Authors' contributions}

SMS, HL designed the article. LMW, SDJ, NL analyzed data. HL, LMW, WJZ wrote the draft manuscript. SMS, HBC, YS revised the manuscript. All authors read and approved the final manuscript.

Funding

SMS has received a grant from the National Natural Science Foundation of China (No. 81972158). For the remaining authors, none were declared funding.

Availability of data and materials

All data used in this study are available in public, and the access policy and procedures are available at: http://charls.pku.edu.cn/index/en.html.

\section{Declarations}

\section{Ethics approval and consent to participate}

The CHARLS was approved by the Ethical Review Committee of Peking University, and all participants signed informed consent at the time of participation. All methods were performed according to the Declaration of Helsinki.

\section{Consent for publication \\ Not applicable.}

\section{Competing interests}

The authors declare no conflict of interest.

\section{Author details}

${ }^{1}$ Peking University School of Nursing, No.38 Xueyuan Road, Haidian District, Beijing, China. ${ }^{2}$ Zhejiang Chinese Medical University School of Nursing, No.548 Binwen Road, Binjiang District, Hangzhou, China. ${ }^{3}$ University of Michigan School of Nursing, No.426 Ingalls Street, Ann Arbor, MI, USA. ${ }^{4}$ Peking University School of Public Health, No. 38 Xueyuan Road, Haidian District, Beijing, China.

${ }^{5}$ Peking University Sixth Hospital, No.51 Hua Yuan Bei Road, Haidian District, Beijing, China. ${ }^{6}$ Research Center of Clinical Epidemiology, Peking University Third Hospital, No.46 Hua Yuan Bei Road, Haidian District, Beijing, China.

Received: 16 September 2021 Accepted: 18 February 2022

Published online: 05 March 2022

\section{References}

1. Cross M, Smith E, Hoy D, Nolte S, Ackerman I, Fransen M, et al. The global burden of hip and knee osteoarthritis: estimates from the global burden of disease 2010 study. Ann Rheum Dis. 2014;73(7):1323-30.

2. GBD 2015 Disease and Injury Incidence and Prevalence Collaborators. Global, regional, and national incidence, prevalence, and years lived with disability for 310 diseases and injuries, 1990-2015: a systematic analysis for the Global Burden of Disease Study 2015. Lancet. 2016;388(10053):1545-602.

3. Deshpande BR, Katz JN, Solomon DH, Yelin EH, Hunter DJ, Messier SP, et al. Number of Persons With Symptomatic Knee Osteoarthritis in the US: Impact of Race and Ethnicity, Age, Sex, and Obesity. Arthritis Care Res (Hoboken). 2016;68(12):1743-50. 
4. Safiri S, Kolahi AA, Smith E, Hill C, Bettampadi D, Mansournia MA, et al. Global, regional and national burden of osteoarthritis 1990-2017: a systematic analysis of the Global Burden of Disease Study 2017. Ann Rheum Dis. 2020;79(6):819-28.

5. Grover AK, Samson SE. Benefits of antioxidant supplements for knee osteoarthritis: rationale and reality. Nutr J. 2016;15:1.

6. GBD 2019 Diseases and Injuries Collaborators. Global burden of 369 diseases and injuries in 204 countries and territories, 1990-2019: a systematic analysis for the Global Burden of Disease Study 2019. Lancet. 2020;396(10258):1204-22.

7. Jung JH, Seok H, Kim JH, Song GG, Choi SJ. Association between osteoarthritis and mental health in a Korean population: a nationwide study. Int J Rheum Dis. 2018;21(3):611-9.

8. Veronese N, Stubbs B, Solmi M, Smith TO, Noale M, Cooper C, et al. Association between lower limb osteoarthritis and incidence of depressive symptoms: data from the osteoarthritis initiative. Age Ageing. 2017;46(3):470-6.

9. Stubbs B, Aluko Y, Myint PK, Smith TO. Prevalence of depressive symptoms and anxiety in osteoarthritis: a systematic review and meta-analysis. Age Ageing. 2016;45(2):228-35.

10. Dell'Isola A, Pihl K, Turkiewicz A, Hughes V, Zhang W, Bierma-Zeinstra S, et al. Risk of comorbidities following physician-diagnosed knee or hip osteoarthritis: a register-based cohort study. Arthritis Care Res (Hoboken). 2021.

11. Swain S, Coupland C, Mallen C, Kuo CF, Sarmanova A, Bierma-Zeinstra SMA, et al. Temporal relationship between osteoarthritis and comorbidities: a combined case control and cohort study in the UK primary care setting. Rheumatology (Oxford). 2021;60(9):4327-39.

12. Gandhi R, Zywiel MG, Mahomed NN, Perruccio AV. Depression and the overall burden of painful joints: an examination among individuals undergoing hip and knee replacement for osteoarthritis. Arthritis. 2015;2015:327161.

13. Marszalek J, Price LL, Harvey WF, Driban JB, Wang C. Outcome expectations and osteoarthritis: association of perceived benefits of exercise with self-efficacy and depression. Arthritis Care Res (Hoboken). 2017;69(4):491-8.

14. Stubbs B, Hurley M, Smith T. What are the factors that influence physical activity participation in adults with knee and hip osteoarthritis? A systematic review of physical activity correlates. Clin Rehabil. 2015;29(1):80-94.

15. Agarwal P, Sambamoorthi U. Healthcare expenditures associated with depression among individuals with osteoarthritis: post-regression linear decomposition approach. J Gen Intern Med. 2015:30(12):1803-11.

16. Vennu V, Misra H, Misra A. Depressive symptoms and the risk of arthritis: A survival analysis using data from the osteoarthritis initiative. Indian J Psychiatry. 2019;61(5):444-50.

17. Zhao Y, Strauss J, Yang G, Giles J, Hu PP, Hu Y, et al. China health and retirement longitudinal study, 2011-2012 national baseline users' guide. Beijing: National School of Development, Peking University; 2013.

18. Liu Q, Wang S, Lin J, Zhang Y. The burden for knee osteoarthritis among Chinese elderly: estimates from a nationally representative study. Osteoarthr Cartil. 2018:26(12):1636-42.

19. The Joint Surgery Branch of the Chinese Orthopaedic Association, the Subspecialty Group of Osteoarthritis, Chinese Association of Orthopaedic Surgeons, the National Clinical Research Center for Geriatric Disorders (Xiangya Hospital), Editorial Office of Chinese Journal of Orthopaedics. Chinese guideline for diagnosis and treatment of osteoarthritis (2021 edition). Chin J Orthop. 2021;41(18):1291-314.

20. Boey KW. Cross-validation of a short form of the CES-D in Chinese elderly. Int J Geriatr Psychiatry. 1999;14(8):608-17.

21. Okely JA, Weiss A, Gale CR. Well-being and arthritis incidence: the role of inflammatory mechanisms. findings from the english longitudinal study of ageing. Psychosom Med. 2017;79(7):742-8.

22. Jin Y, Luo Y, He P. Hypertension, socioeconomic status and depressive symptoms in Chinese middle-aged and older adults: Findings from the China health and retirement longitudinal study. J Affect Disord. 2019;252:237-44

23. National Bureau of Statistics. Statistics Bureau announced: National urban and rural residents' income growth in 2011 in China. 2012. Available from: http://www.gov.cn/gzdt/2012-01/20/content_2050056.htm.
24. Bai A, Tao L, Huang J, Tao J, Liu J. Effects of physical activity on cognitive function among patients with diabetes in China: a nationally longitudinal study. BMC Public Health. 2021;21(1):481.

25. Craig CL, Marshall AL, Sjostrom M, Bauman AE, Booth ML, Ainsworth BE, et al. International physical activity questionnaire: 12-country reliability and validity. Med Sci Sports Exerc. 2003;35(8):1381-95.

26. Katz S, Ford AB, Moskowitz RW, Jackson BA, Jaffe MW. Studies of illness in the aged. The index of ADL: a standardized measure of biological and psychosocial function. JAMA. 1963;185:914-9.

27. Lawton MP, Lawton MP, Brody EM, Brody EM. Assessment of older people: Self-maintaining and instrumental activities of daily living. Gerontologist. 1969;9(3):179-86.

28. Kessler RC, Berglund P, Demler O, Jin R, Koretz D, Merikangas KR, et al. The epidemiology of major depressive disorder: results from the National Comorbidity Survey Replication (NCS-R). JAMA. 2003;289(23):3095-105.

29. Rathbun AM, Shardell MD, Ryan AS, Yau MS, Gallo JJ, Schuler MS, et al. Association between disease progression and depression onset in persons with radiographic knee osteoarthritis. Rheumatology (Oxford). 2020

30. Shang X, Peng W, Hill E, Szoeke C, He M, Zhang L. Incidence of medication-treated depression and anxiety associated with long-term cancer, cardiovascular disease, diabetes and osteoarthritis in communitydwelling women and men. EClinicalMedicine. 2019;15:23-32.

31. Unutzer J. Clinical practice. Late-life depression. N Engl J Med. 2007;357(22):2269-76.

32. Akintayo RO, Yerima A, Olaosebikan HB, Uhunmwangho C, Akpabio AA. How much gloom is in groans? Depression and its determinants in Nigerian patients with knee osteoarthritis: a multi-center cross-sectional study. Clin Rheumatol. 2019;38(7):1971-8.

33. Wilkie R, Blagojevic-Bucknall M, Jordan KP, Lacey R, McBeth J. Reasons why multimorbidity increases the risk of participation restriction in older adults with lower extremity osteoarthritis: a prospective cohort study in primary care. Arthritis Care Res (Hoboken). 2013;65(6):910-9.

34. Thomas S, Browne H, Mobasheri A, Rayman MP. What is the evidence for a role for diet and nutrition in osteoarthritis? Rheumatology (Oxford). 2018;57(suppl_4):iv61-74.

35. Sarris J, O’Neil A, Coulson CE, Schweitzer I, Berk M. Lifestyle medicine for depression. BMC Psychiatry. 2014;14:107.

36. Milaneschi Y, Hoogendijk W, Lips P, Heijboer AC, Schoevers R, van Hemert $A M$, et al. The association between low vitamin $D$ and depressive disorders. Mol Psychiatry. 2014;19(4):444-51.

37. Joseph GB, McCulloch CE, Nevitt MC, Neumann J, Lynch JA, Lane NE, et al Associations between Vitamin C and D Intake and Cartilage Composition and Knee Joint Morphology over 4 years: Data from the Osteoarthritis Initiative. Arthritis Care Res (Hoboken). 2019.

38. Mathiessen A, Conaghan PG. Synovitis in osteoarthritis: current understanding with therapeutic implications. Arthritis Res Ther. 2017;19(1):18.

39. da Silva MR, Linhares D, Vasconcelos DM, Alves CJ, Neves N, Costa G, et al. Neuroimmune expression in hip osteoarthritis: a systematic review. BMC Musculoskelet Disord. 2017:18(1):394.

40. O'Donovan A, Rush G, Hoatam G, Hughes BM, McCrohan A, Kelleher $C$, et al. Suicidal ideation is associated with elevated inflammation in patients with major depressive disorder. Depress Anxiety. 2013:30(4):307-14.

41. Lespasio MJ, Piuzzi NS, Husni ME, Muschler GF, Guarino A, Mont MA. Knee Osteoarthritis: A Primer. Perm J. 2017;21:16-183.

42. Mcllvane JM, Schiaffino KM, Paget SA. Age differences in the pain-depression link for women with osteoarthritis. Functional impairment and personal control as mediators. Womens Health Issues. 2007;17(1):44-51.

43. Moise N, Khodneva Y, Jannat-Khah DP, Richman J, Davidson KW, Kronish $I M$, et al. Observational study of the differential impact of time-varying depressive symptoms on all-cause and cause-specific mortality by health status in community-dwelling adults: the REGARDS study. BMJ Open. 2018;8(1):e017385.

44. Colman I, Kingsbury M, Sucha E, Horton NJ, Murphy JM, Gilman SE. Depressive and anxious symptoms and 20-year mortality: Evidence from the Stirling County study. Depress Anxiety. 2018;35(7):638-47.

45. Gallo JJ, Anthony JC, Muthen BO. Age differences in the symptoms of depression: a latent trait analysis. J Gerontol. 1994;49(6):P251-64.

46. National Institute for Health and Clinical Excellence. Osteoarthritis: Care and Management in Adults. London; 2014. 
47. Abbasi J. Can Exercise Prevent Knee Osteoarthritis? JAMA 2017;318(22):2169-71

48. Briani RV, Ferreira AS, Pazzinatto MF, Pappas E, De Oliveira SD, Azevedo FM. What interventions can improve quality of life or psychosocial factors of individuals with knee osteoarthritis? A systematic review with metaanalysis of primary outcomes from randomised controlled trials. $\mathrm{Br} J$ Sports Med. 2018;52(16):1031-8.

49. Atlantis E, Fahey P, Cochrane B, Smith S. Bidirectional associations between clinically relevant depression or anxiety and COPD: a systematic review and meta-analysis. Chest. 2013;144(3):766-77.

50. Nouwen A, Adriaanse MC, van Dam K, Iversen MM, Viechtbauer W, Peyrot $M$, et al. Longitudinal associations between depression and diabetes complications: a systematic review and meta-analysis. Diabet Med. 2019;36(12):1562-72.

51. Tang B, Yuan S, Xiong Y, He Q, Larsson SC. Major depressive disorder and cardiometabolic diseases: a bidirectional Mendelian randomisation study. Diabetologia. 2020;63(7):1305-11.

\section{Publisher's Note}

Springer Nature remains neutral with regard to jurisdictional claims in published maps and institutional affiliations.

- fast, convenient online submission

- thorough peer review by experienced researchers in your field

- rapid publication on acceptance

- support for research data, including large and complex data types

- gold Open Access which fosters wider collaboration and increased citations

- maximum visibility for your research: over $100 \mathrm{M}$ website views per year

At BMC, research is always in progress.

Learn more biomedcentral.com/submissions 